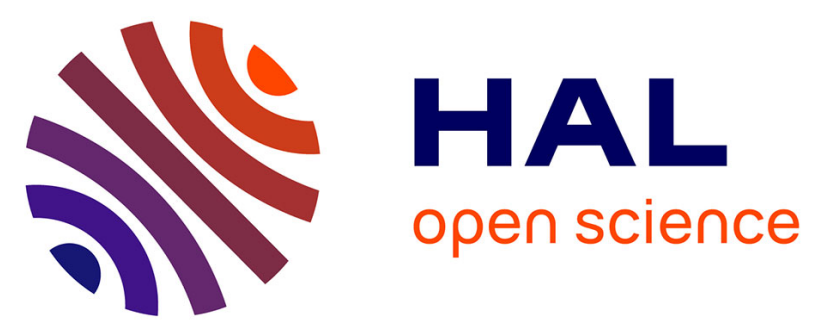

\title{
A proposal on frequency management methodologies for WCDMA systems using cell coupling matrices
}

\author{
Jad Nasreddine, Jordi Perez Romero, Oriol Sallent, Ramon Agusti, Xavier
}

Lagrange

\section{- To cite this version:}

Jad Nasreddine, Jordi Perez Romero, Oriol Sallent, Ramon Agusti, Xavier Lagrange. A proposal on frequency management methodologies for WCDMA systems using cell coupling matrices. VTC 2006: 64th IEEE Vehicular Technology Conference, Sep 2006, Montréal, Canada. pp.1 - 5, 10.1109/VTCF.2006.271 . hal-02345908

\section{HAL Id: hal-02345908 \\ https://hal.science/hal-02345908}

Submitted on 4 Nov 2019

HAL is a multi-disciplinary open access archive for the deposit and dissemination of scientific research documents, whether they are published or not. The documents may come from teaching and research institutions in France or abroad, or from public or private research centers.
L'archive ouverte pluridisciplinaire HAL, est destinée au dépôt et à la diffusion de documents scientifiques de niveau recherche, publiés ou non, émanant des établissements d'enseignement et de recherche français ou étrangers, des laboratoires publics ou privés. 


\title{
A Proposal on Frequency Management Methodologies for WCDMA Systems using Cell Coupling Matrices
}

\author{
J. Nasreddine ${ }^{(1)}$, J. Pérez-Romero $^{(1)}$, O. Sallent ${ }^{(1)}$, R. Agusti $^{(1)}$, X. Lagrange ${ }^{(2)}$ \\ ${ }^{(1)}$ Dept. TSC, Universitat Politècnica de Catalunya (UPC), c/ Jordi Girona, 1-3, Campus Nord, 08034 Barcelona, Spain \\ email: [jnassred, jorperez, sallent, ramon]@tsc.upc.edu \\ ${ }^{(2)}$ Dept. RSM, GET/ENST de Bretagne, 2 rue de la châtaigneraie, CS 17607, 35576 Cesson Sévigné Cedex, France \\ email: xavier.lagrange@enst-bretagne.fr
}

\begin{abstract}
Although a single carrier frequency is usually considered in Wideband Code Division Multiple Access (WCDMA) systems, each operator has more than one carrier frequency in practical 3G systems. Moreover, QoS levels and the throughput within a given frequency highly depend on interference patterns, which are mainly related to cell-frequency allocation. Therefore, frequency management plays a key role in WCDMA network planning. However, the frequency management problem has not been in the center of attention for WCDMA systems so far due to the fact that WCDMA has not yet been implemented in wide range with all possible services. Nevertheless, the arrival of multimedia services will emphasize the critical importance of a smart frequency allocation. In this context, the presence of several frequencies for each operator (typically 2 or 3 in Europe) manifests itself as a fertile dimension of flexibility to be exploited. This paper introduces a novel frequency management methodology based on coupling matrices that reflects the interaction between cells for a given interference pattern. The proposed methodology is implemented as an integral task of the planning tool and has lead to better results than the frequency allocation in the classical hierarchical cell structure.
\end{abstract}

\section{INTRODUCTION}

In WCDMA systems, several users share the same carrier frequency using different code sequences, which have good autocorrelation and cross-correlation characteristics. However, the lack of synchronization and the impact of multipath reduce the efficiency of these sequences and lead to the appearance of intra-cell and inter-cell interferences. These interferences are the main limiting factors for WCDMA capacity. Hence, interference reduction is a vital task in WCDMA systems and can be achieved by combining radio network planning and Radio Resource Management (RRM) techniques that can distribute efficiently the scarce radio resource units. A resource unit in WCDMA systems can be seen as the combination of a carrier frequency, a spreading code and the power dedicated to this pair. The transmission power allocation and the distribution of spreading codes have been widely studied in the literature using different RRM techniques such as power control, scheduling, call admission control, etc [1]. On the contrary, and even though each WCDMA operator has several carrier frequencies (typically 2 or 3 in Europe), the Frequency Management Problem (FMP) in WCDMA has hardly been addressed so far in the open literature to the authors' best knowledge. The introduction of the frequency dimension into planning and RRM problems provides an additional degree of flexibility to reduce the interference in each carrier and increase system capacity, thus becoming an important aspect to be developed for a fruitful exploitation of the inherent WCDMA potentials.

Available scarce research works relate the FMP in WCDMA to Hierarchical Cell Structures (HCS) scenarios, mainly by analyzing the feasibility to operate macro-cell and micro-cell layers with the same frequency, then breaking the approach by allocating different frequencies to the two layers [2]-[5]. Nevertheless, the FMP neither has to be limited to the HCS nor it has to be presumed that it has a priori guide on a suitable solution, since it is strongly dependant on interference and traffic distribution along the deployed scenario.

The FMP was widely studied in TDMA/FDMA systems and involved two procedures, namely the design of an appropriate representation of radio constraints and the design of optimization algorithms. Radio constraints include all types of interaction between equipments and they are captured in the so-called compatibility matrix [6][7]. Once radio constraints are captured, optimization algorithms provide frequency allocation that maximizes the capacity while satisfying QoS constraints [8]-[12].

Existing methodologies for TDMA/FDMA systems are substantially different from the methodologies envisaged in this paper for WCDMA systems due to the different nature of constraints imposed by the radio interface. In fact, cell interaction in TDMA/FDMA systems depends only on channel characteristics between the two cells due to the presence of one transmission in each cell and a primitive power control. Hence, the estimation of cell interaction is generally simple and has been widely investigated [6][7]. However, the representation of radio constraints in WCDMA systems cannot be extracted with the same simplicity as in TDMA/FDMA due to the existence of intra-cell and inter-cell interference together with fast power control schemes leading to a high correlation between interference profiles, cell loads, mobile positions, etc. This complexity may lead to a questionable representation of cell interaction if the same model as TDMA/FDMA systems is used. Therefore, a specific representation for radio constraints has to be designed to fit WCDMA characteristics. In that sense, 
the authors of [13] presented a methodology based on load gradient computations which revealed its suitability to capture the interaction among cells and was identified as a potential candidate to be used for frequency management in WCDMA.

Taking into account the above framework, this paper addresses the FMP in WCDMA systems as an integral task of an advanced $3 \mathrm{G}$ planning tool. The paper considers the WCDMA deployment phase in which traffic requirements are such that not all the available carriers are fully loaded, so that the practical availability of several carriers per operator can be exploited to improve interference patterns. Consequently, system performance can be ameliorated by means of the proposed frequency management strategies. Clearly, in the extreme situation of full load in all carriers, a frequency reuse of one (i.e. all carriers allocated in all base stations) would be the most adequate solution. Accordingly, the particular scope of the paper is the situation in which a single carrier is required per base station, although it is thought that the proposed methodology could be extended to cope with the case in which more than one carrier is required in some base stations depending on traffic patterns. Based on the general two-step procedure used in TDMA/FDMA systems, the proposed methodology covers first the definition of a coupling matrix whose entries capture the degree of interaction among the different cells (i.e. an equivalent concept to the compatibility matrix) using radio network measurements such as $E_{b} / N_{0}$, interference and pathloss. Only very recently, there have been few attempts to design coupling matrices for WCDMA systems. In [14], the authors have proposed a coupling matrix assuming that the received interference of each cell can be easily isolated, which is not so explicit. In addition to the fact that our contribution proposes solutions for the whole FMP, a relevant novelty included hereinafter is that we propose a methodic scheme to capture the coupling matrix.

In the second step, the coupling matrix is used as the input to a heuristic algorithm that provides the frequency assignment. In this case, the lower number of available frequencies in WCDMA systems when compared to TDMA/FDMA systems leads to a simplification in the heuristic algorithm design.

The proposed methodology considers only the uplink and can be extended to take into account the downlink by using the model developed in [1]. Furthermore, it is worth mentioning that the methodology can be applicable both at the initial static radio network planning phase and in operational systems in order to cope with significant changes in the distribution of cell loads during time, thus envisaging dynamic frequency planning mechanisms (i.e. frequency assignment to cells may vary along time and space) at a rather long-term scale (i.e. once or twice in a day, several times in a week, etc.). In this case, the dynamic mechanisms will be able to cope with sudden but, at the same time, significant and long lasting traffic variations, which can be of high relevance in practical WCDMA networks.

This paper is organized as follows: In section II, we develop an analytical framework for a coupling matrix and we propose a simple heuristic optimization algorithm to solve the FMP. In section III, we introduce the simulation model, which is used to obtain the results presented in section IV. Finally, we conclude with relevant remarks and future works.

\section{FREQUENCY MANAGEMENT METHODOLOGY}

Let us consider a well dimensioned WCDMA system of $K$ base stations and $F$ frequencies. Hence, all users' $E_{b} / N_{0}$ s are around the required thresholds.

\section{A. Coupling Matrix based on Load Gradient}

Coupling matrices are intended to reflect interaction between different cells. In other words, entry $C_{j, l}$ of coupling matrix $\boldsymbol{C}$ represents the impact of cell $l$ on cell $j$. Hereafter, we propose an analytical framework to derive a suitable representation of cell interactions based on load gradient computation. In the coupling matrix based on load gradient, the entry $C_{j, l}$ is computed using the derivative of the load factor in cell $j$ with respect to the load factor in cell $l\left(\partial \eta_{j} / \partial \eta_{l}\right)$, which reflects the impact of load factor variation in cell $l$ on load factor variation in cell $j$. We remind the reader that the load factor of cell $j$ in uplink is given by the following equation:

$$
\eta_{j}=1-\frac{P_{N}}{P_{R, j}+\chi_{j}+P_{N}}
$$

where $P_{N}$ is the background noise power, $P_{R, j}$ is the total own cell power received by cell $j$ and $\chi_{j}$ is the inter-cell interference experienced by cell $j$. The $E_{b} / N_{0}$ of mobile $i_{j}$ is given by:

$$
\left(\frac{E_{b}}{N_{0}}\right)_{i_{j}}=\frac{P_{j, i_{j}} \Theta_{i_{j}}}{\left[P_{R, j}-P_{j, i_{j}}\right]+\chi_{j}+P_{N}}
$$

where $P_{j, i_{j}}$ is the useful received power by base station $j$ from mobile $i_{j}$ and $\Theta_{i_{j}}$ is the transmission spreading factor of mobile $i_{j}$. Using the load factor equation from [1], we can write:

$$
\eta_{j}=\frac{S_{j, j}^{U L}+\sum_{\substack{k=1 \\ k \neq j}}^{K} \frac{S_{k, j}^{U L}}{1-\eta_{k}}}{1+\sum_{\substack{k=1 \\ k \neq j}}^{K} \frac{S_{k, j}^{U L}}{1-\eta_{k}}}
$$

where:

$$
S_{k, j}^{U L}=\sum_{i_{k}=1}^{n_{k}} \frac{L_{i_{k}, k}}{L_{i_{k}, j}} \frac{1}{\frac{\Theta_{i_{k}}}{\left(\frac{E_{b}}{N_{o}}\right)_{i_{k}}}+1}
$$

where $L_{i_{k}, j}$ is the pathloss of mobile $i_{k}$ towards base station $j$ and $n_{k}$ is the number of users in cell $k$. Thus, we can write:

$$
\frac{\partial \eta_{j}}{\partial \eta_{l}}=B_{j} \sum_{\substack{k=1 \\ k \neq j}}^{K} \frac{S_{k, j}^{U L}}{\left(1-\eta_{k}\right)^{2}} \frac{\partial \eta_{k}}{\partial \eta_{l}}
$$

where $B_{j}$ is given by:

$$
B_{j}=\frac{1-S_{j, j}^{U L}}{\left(1+\sum_{\substack{k=1 \\ k \neq j}}^{K} \frac{S_{k, j}^{U L}}{1-\eta_{k}}\right)^{2}}
$$

Furthermore, we define the sum $J_{j}$ by the sum of the gradient vector related to cell $j$ and given by the following relation: 


$$
J_{j}=\sum_{m=1}^{K} \frac{\partial \eta_{j}}{\partial \eta_{m}}
$$

The term $J_{j}$ reflects the impact of load factor variations in all cells on load factor variation in cell $j$. It must be noted here, that the factor $\partial \eta_{j} / \partial \eta_{j}$ is included in the sum to have a convenient final expression of $J_{j}$. The value of this term is a constant (i.e. 1); therefore, it does not have any impact.

After sum computation, we find:

$$
J_{j}=B_{j} \sum_{\substack{l=1 \\ l \neq j}}^{K} \frac{S_{l, j}^{U L}}{\left(1-\eta_{l}\right)^{2}} \sum_{m=1}^{K} \frac{\partial \eta_{l}}{\partial \eta_{m}}
$$

Using equation (7) for all cells, we find:

$$
J_{j}=B_{j} \sum_{\substack{l=1 \\ l \neq j}}^{K} \frac{S_{l, j}^{U L}}{\left(1-\eta_{l}\right)^{2}} J_{l}
$$

Each element of the right term can be seen as the contribution of a neighboring load factor variation to the total variation of load factor in cell $j$. Thus, the coefficient of element $l$ represents the impact of cell $l$ on cell $j$. Using these elements, we can define an intermediate coupling matrix $\boldsymbol{A}$ :

$$
A_{j, l}=\left\{\begin{array}{cc}
1 & \text { if } l=j \\
B_{j} \frac{S_{l, j}^{\mathrm{UL}}}{\left(1-\eta_{l}\right)^{2}} & \text { otherwise }
\end{array}\right.
$$

This matrix considers only the variation of the load factor. However, the cell load has also a significant impact on the interaction with other cells. In order to take into account this effect, we multiply each column by the corresponding cell load. Then the final coupling matrix $\boldsymbol{C}$ can be written as:

$$
C=A L
$$

where $\boldsymbol{L}$ is a diagonal matrix whose entries are defined by:

$$
L_{j, j}=\sum_{i_{j}=1}^{n_{j}} R_{i_{j}}
$$

where $R_{i_{j}}$ is mobile $i_{j}$ data rate at physical layer in Kbits/s.

In order to consider the mutual interaction between two cells, the following symmetric matrix is defined using the asymmetric matrix $\boldsymbol{C}$ :

$$
\boldsymbol{O}=\boldsymbol{C}+\boldsymbol{C}^{t}
$$

where $\boldsymbol{C}$ is the transpose of $\boldsymbol{C}$. Hence, each entry $O_{j, k}$ combines the impact of cell $j$ on cell $k$ and the impact of cell $k$ on cell $j$.

\section{B. Heuristic Optimization Algorithm}

The objective of the optimization algorithm is to minimize the total outage probability in the system, which is defined as the ratio of unsatisfied users to the total number of users. A user is considered unsatisfied if its $E_{b} / N_{0}$ is lower than the target $E_{b} / N_{0}$. Notice that the outage probability has been considered here as a representative QoS metric taking into account its relationship with other QoS metrics (e.g. a high outage probability will be associated with a high error rate for real time services and with a high delay for non real time services). Moreover, the total outage probability in WCDMA systems is highly related to interference which in turn is related to the coupling matrix. Consequently, our objective is to minimize the interference profile using an offline heuristic algorithm that reduces cell interactions.

Herein, we define $X_{f}$ as the set of cells operating with frequency $f$. These sets are initially empty sets.

Using matrix $\boldsymbol{O}$, a heuristic algorithm that tends to avoid high interactions among cells is defined by the following steps:

1. Cells are sorted using the sum of the corresponding rows in matrix $\boldsymbol{O}$.

2. The $F$ frequencies are allocated with one-to-one association to the first $F$ cells with highest sum and sets $X_{f}$ are updated.

3 . For the next cell $i$ of the remaining sorted cells, define the set of variables $\left\{\rho_{f}\right\}_{f=1 . . F}$ by:

$$
\rho_{f}=\sum_{k \in X_{f} \cup\{i\}} S_{i, k}
$$

4. Thereafter, cell $i$ is associated to frequency $f_{c}$ such that:

$$
\rho_{f_{c}}=\min _{f \in\{1, \ldots, F\}} \rho_{f}
$$

\section{Add cell $i$ to set $X_{f}$. and repeat from step 3}

In each step, the algorithm reduces the impact of introducing a new cell on the existing cells and the impact of the latter on the new cell. At the beginning, frequencies are allocated to cells with the highest interference contribution because these cells have the highest impact on interference patterns. The remaining cells are then distributed such as the total cell interaction is minimized. Using this method, we can avoid the allocation of the same frequency to cells with high interaction.

\section{SIMULATION MODEL}

The performance of the proposed frequency management methodology has been evaluated by means of Monte Carlo simulations in a scenario with 37 macro-cells and a set of micro-cells. Each simulation consists of 10000 snapshots. In the simulation, each mobile is connected to the base station with the highest $E_{d} / N_{0}$ (signal energy per chip over Noise power spectral density) of the downlink pilot channel. Simulation parameters are summarized in table I. In order to study the performance of the proposed scheme, the traditional HCS is considered as a reference scheme for comparison purposes. In the HCS, macro-cells share the same frequency, while micro-cells (when they exist) share the second one. The simulations have been carried out with four different representative case studies. In the first three cases, a first traffic layer is uniformly distributed and a second layer including dense hotspots is added. Case study 1 corresponds to the first phase of WCDMA system deployment where hotspots start to appear, whereas no micro-cells are implemented. Consequently, only one frequency is used by the traditional HCS. The cell layout is the same as the layout shown in figure 2 without micro-cells. In case study 2, each hotspot is served by a micro-cell in the center of the hotspot (figure 2). In case study 3 , the micro-cells are not at the center of the hotspots (figure 3). In case study 4, there are neither hotspots nor microcells, but each macro-cell has a different traffic density (figure 4). In each case study of the first three cases, three layouts, denoted as layout 1, 2 and 3 respectively, are considered with five, eight and ten hotspots with the corresponding micro-cells. 
The micro-cell, when exists, is placed at the intersections of three cells depending on the layout (figure 2).

\section{RESULTS}

In this section, we present the results obtained using the different case studies and layouts. First, we show in figure 1 several values of matrix $\boldsymbol{O}$ from layout 2 of case study 2 . We remind that the entries of matrix $\boldsymbol{O}$ are used by variables $\rho_{f}$. From this example we can see that the matrix efficiently reflects the interaction between cells. For instance, the interaction between micro-cells is very low (0.03) because mobiles in these cells have very low pathloss toward their servers and very high pathlosses toward other base stations. However, the interaction between micro-cells and neighboring macro-cells is very high (84). Indeed, some mobiles that are geographically near to micro-cells are connected to a macrocell due to the difference in pilot power. Therefore, these mobiles introduce high interferences into micro-cells. Moreover, the interaction between macro-cells is higher when the macro-cells are close. Thus, the proposed coupling matrix takes into account both cell loads and geographical positions.

In figures 2, 3 and 4, we show the frequency configurations for case studies 2, 3 and 4 when the proposed methodology is used. Herein, cells with high loads (i.e. generally cells with high entries in the coupling matrix), tend to not share the same frequency with more than one neighboring cell. Moreover, the proposed method tends to allocate the same frequency to a set of neighboring cells when it is possible, which gives users the flexibility of smooth mobility without changing the frequency during each handover.

In figure 5, we show the total outage probability in the system when the HCS and the frequency management methodology are used for the first three case studies. As we can see, the proposed methodology reduces the outage probability by more than $40 \%$ in all cases. Moreover, the slope of the outage probability as a function of the number of hotspots (i.e. the different layouts) is smaller when the proposed methodology is used, especially when the micro-cells are not at the center of the hotspots. This is due to the fact that the proposed methodology is slightly dependent on the number of hotspots in contrary to the HCS. In the fourth case study, the outage probability decreases from $5.8 \%$ when HCS is used to $0.3 \%$ when the proposed scheme is used. In all cases, the traditional HCS appear to be unable to offer an acceptable assignment and an appropriate solution without adding more base stations. In contrast, the proposed methodology manifests as an elegant solution to solve this problem only by changing the frequency allocation. In brief, this solution will reduce the cost of operator infrastructure. It should be emphasized here that the optimization algorithm complexity is a linear function of the number of cells. Therefore, its convergence is very fast and can be easily performed in operational systems even if a large set of cells are considered.

\section{CONCLUSIONS AND FUTURE WORKS}

In this paper, we have proposed a novel formulation of the interaction between cells, using load factor gradients for the uplink in WCDMA systems. This formulation is used to design an efficient frequency management methodology using a heuristic algorithm. Simulations results have shown that the obtained coupling matrix reflects efficiently cell interactions. Furthermore, the proposed algorithm drastically decreases the outage probability when compared to the traditional allocation according to HCS. The proposed scheme is an offline scheme at this stage. However, an online scheme that can cope with relatively fast variations in the system can be developed thanks to the interesting characteristics of the proposed matrix.

\section{ACKNOWLEDGEMENTS}

This work is partially funded by the European Network of Excellence NEWCOM (Contract number 507325), and by the COSMOS grant (ref. TEC2004-00518, Spanish Ministry of Science and Education and European Regional Development Fund).

\section{REFERENCES}

[1] J. Pérez-Romero, O. Sallent, R. Agusti, M. A. Diaz-Guerra, Radio Resource Management strategies in UMTS, John wiley \& Sons, 2005

[2] X. Lagrange, "Multitier Cell Design," IEEE Communications Magazine, Vol. 35, $\mathrm{N}^{\circ} 8,1997$, pp. 60-64

[3] S.A. Ghorashi, H.K. Cheung, F. Said, A.H Aghvami, "Performance of a CDMA-based HCS network with hybrid speed/overflow-sensitive handover strategy," IEE Proc.-Communications, vol. 150, $\mathrm{N}^{\circ} 4,2003$, pp. $293-297$

[4] T. Rautiainen, "Breaking the Hierarchical Cell Structure in WCDMA Networks", Proc. of the 55th IEEE VTC Spring conference, 2002

[5] R. S., Karlsson, "Sharing radio resources in hierarchical cell structures utilizing slow frequency hopping," Proc. of the $49^{\text {th }}$ IEEE Vehicular Technology Conference, vol. 1, 1999, pp. 412-416

[6] J. Abril, F. Comellas, A.Cortes, J. Ozon, M. Vaquer, A multiagent system for frequency assignment in cellular radio networks, IEEE Transactions on Vehicular Technology, Vol. 49, $\mathrm{N}^{\circ} 5,2000$, pp. $1558-1565$

[7] M.D. Anton, P. Koch, D. Kunz, B. Ruber, M. Ullrich, "An adaptive method to learn the compatibility matrix for microcellular systems," Proc. of the 44th IEEE VTC spring conference, Vol. 2, 1994, pp. 848-852

[8] D. Kunz, "Channel assignment for cellular radio using neural network," IEEE Transactions on Vehicular Technology, Vol. 40, 1991, pp. 188-193

[9] A. Gamst and W. Rave, "On frequency assignment in mobile automatic telephone systems," Proc. GLOBECOM'82, 1982, pp. 309-315

[10] Z. He, Y. Zhang, C. Wei, and J. Wang, "A multistage self-organizing algorithm combined transiently chaotic neural network for cellular channel assignment," IEEE Transactions on Vehicular Technology, vol. 51, 2002, pp. 1386- 1396

[11] R.-H. Cheng, C.-W. Yu, and T.-K. Wu, "A Novel Approach to the Fixed Channel Assignment Problem," Journal of Information Science and Engineering, vol. 21, 2005, pp. 39-58

[12] G. Kendall and M. Mohamad, "Channel Assignment Optimisation Using a Hyper-heuristic," Proc. of the IEEE Conference on Cybernetics and Intelligent Systems, 2004

[13] J. Pérez-Romero, O. Sallent, R. Agusti "A Novel Framework for Robust WCDMA Planning under Changing Spatial Traffic Distributions", accepted in VTC Spring 2006.

[14] S. Ben Jamaa, H. Dubreil, Z. Altman, and A. Ortega, "Quality Indicator Matrices and Their Contribution to WCDMA Network Design", IEEE Transactions on Vehicular Technology, Vol. 54, No. 3, May 2005

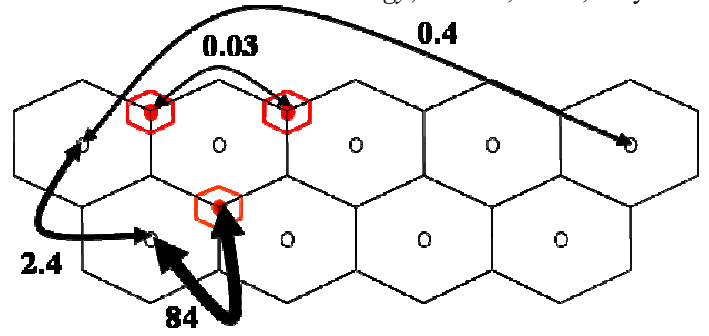

Figure 1. The entries of matrix $\boldsymbol{O}$ in case study 2 and layout 2 


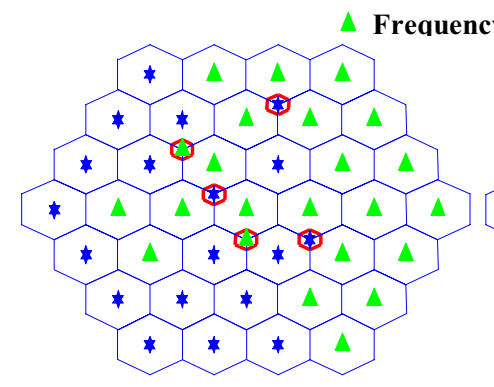

Layout 1

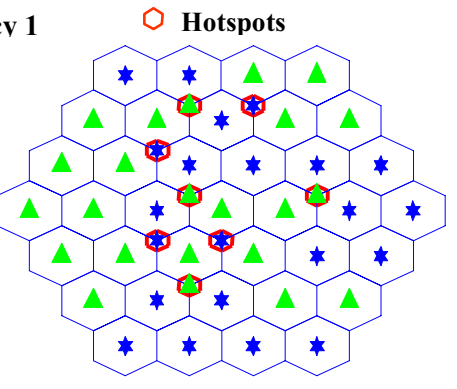

Layout 2
* Frequency 2

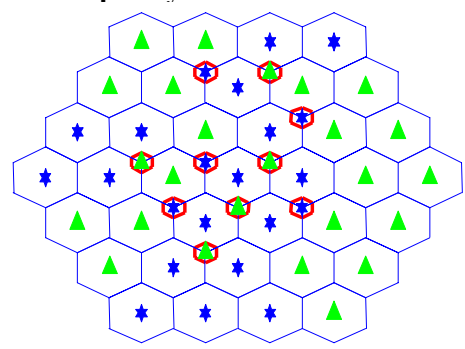

Layout 3

Figure 2. The three cell layouts and corresponding frequency allocation using the proposed coupling matrix for case study 2

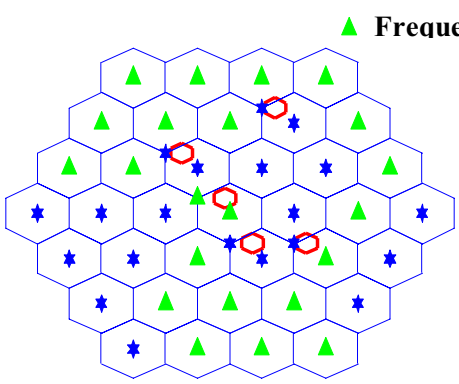

Layout 1

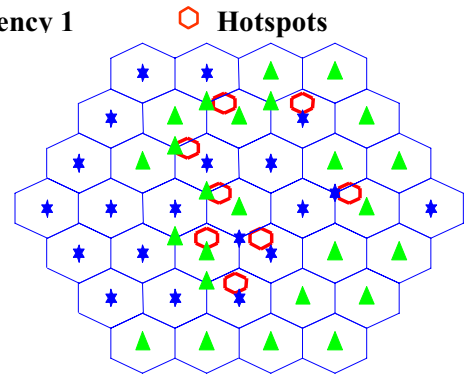

Layout 2

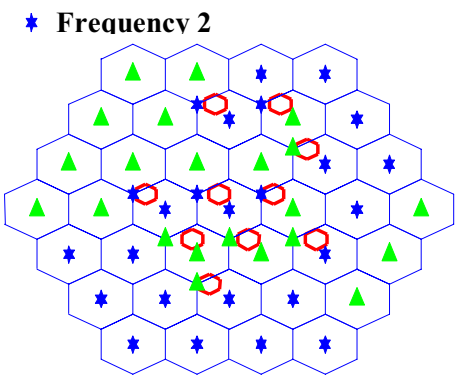

Layout 3

Figure 3. The three cell layouts and corresponding frequency allocation using the proposed coupling matrix for case study 3

TABLE I.

SIMULATION PARAMETERS

\begin{tabular}{|l|l|}
\hline \multicolumn{2}{|c|}{ Macro-cell layer } \\
\hline BS pilot power & $30 \mathrm{dBm}$ \\
\hline Cell radius & $0.6 \mathrm{~km}$ \\
\hline \multicolumn{2}{|c|}{ Cotspot layer } \\
\hline Micro-cell BS pilot power & $12 \mathrm{dBm}$ \\
\hline Cell radius & $0.2 \mathrm{Km}$ \\
\hline \multicolumn{2}{|c|}{ Common parameters } \\
\hline Number of frequencies & 2 \\
\hline Pathloss model & $128.1+37.6 \log _{10} \mathrm{~d}(\mathrm{Km})$ \\
\hline Background noise & $-103 \mathrm{dBm}$ \\
\hline UE maximum transmitted power & $21 \mathrm{dBm}$ \\
\hline UE minimum transmitted power & $-44 \mathrm{dBm}$ \\
\hline $\mathrm{E}_{\mathrm{b}} / \mathrm{N}_{0}$ target & $4 \mathrm{~dB}$ \\
\hline Spreading factor & 256 \\
\hline Shadowing factor deviation & $10 \mathrm{~dB}$ \\
\hline Shadowing factor crosscorrelation & 0.5 \\
\hline Power control & Perfect power control \\
\hline
\end{tabular}

- Hierarchical Cell Structure

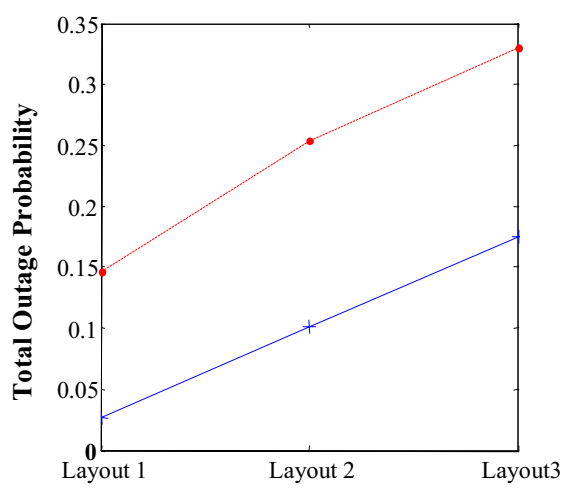

Case study 1 (mobile densities are 20 and 400 mobiles $/ \mathrm{km}^{2}$ respectively in the scenario and hotspots)

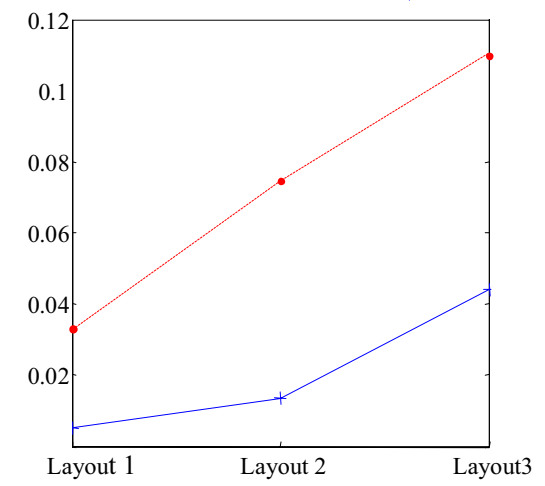

study 2 (mobile densities are 25 and 500 mobil
respectively in the scenario and hotspots)

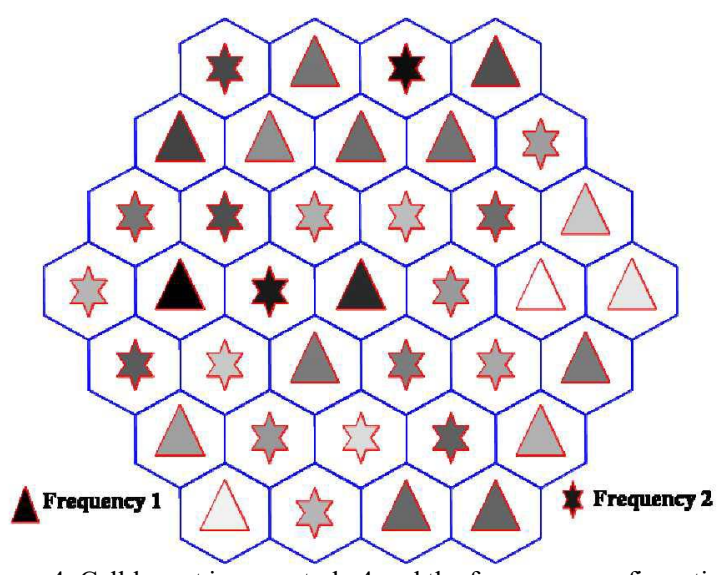

Figure 4. Cell layout in case study 4 and the frequency configuration using the proposed method (the gray level reflects the values of cell loads)

Figure 5. The total outage probability for the first three case studies and the three layouts

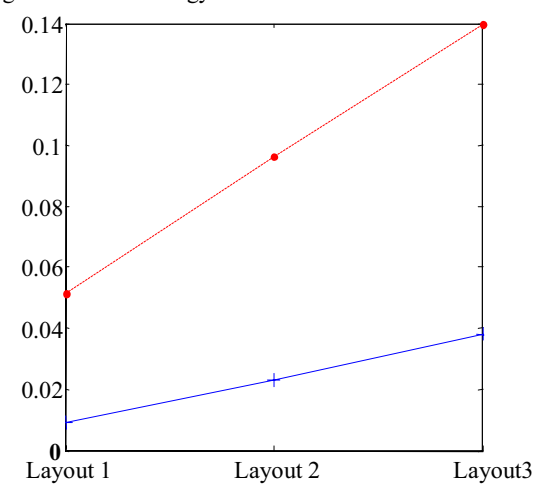

$\mathrm{m}^{2}$ Case study 2 (mobile densities are 20 and 300 mobiles $/ \mathrm{km}^{2}$ respectively in the scenario and hotspots) 